\title{
Determinación de ecuaciones alométricas para estimar biomasa y carbono en Pinus patula Schl. et Cham
}

\author{
Rosalino Díaz-Franco ${ }^{1}$, Miguel Acosta-Mireles ${ }^{2}$, \\ Fernando Carrillo-Anzures ${ }^{2}$, Enrique Buendía-Rodríguez ${ }^{2}$, \\ Eulogio Flores-Ayala ${ }^{2}$ y Jorge D. Etchevers-Barra ${ }^{3}$
}

\section{RESUMEN}

En el Ejido Mariano de Matamoros, Tlaxco, Tlaxcala, se seleccionaron 25 árboles de Pinus patula Schl. et Cham., y se determinaron su biomasa y carbono en el estrato aéreo para ajustar el modelo $Y=b^{*} X^{k}$, tomando como variable independiente $(X)$ el diámetro normal (DN). Se obtuvieron muestras de cada uno de los siguientes componentes (fuste, ramas y follaje) de cada árbol, y en el campo se determinó el peso fresco, tanto de la muestra como del componente, Las muestras se llevaron al laboratorio para ser secadas y obtener su peso seco, para después estimar la biomasa total de cada árbol, usando para ello la relación peso seco: peso fresco de las muestras. Para determinar el carbono de los diferentes componentes se tomaron muestras de cinco árboles y fueron analizadas en el laboratorio, obteniendo asi $52,21 \%$ de carbono en el follaje, $49,47 \%$ en las ramas y $49,26 \%$ en el fuste, de esta forma la concentración promedio por árbol fue de 50,31\%. Para estimar la biomasa se ajustó y generó la ecuación $B=0.0357^{*} D N^{2.6916}$, y para estimar el carbono la ecuación ajustada fue $C C=0.021{ }^{*} D N^{2.6451}$, con los coeficientes de determinación para ambas de 0,98.

PALABRAS CLAVE:

alometría, biomasa, carbono, ecuación, Pinus patula.

\begin{abstract}
In the community of "Mariano de Matamoros", Tlaxco, Tlaxcala, 25 trees of Pinus patula Schl. et Cham., were selected and aboveground biomass and carbon contents were determined to fit the model $Y=b^{*} X^{k}$, using diameter at breast height as an independent variable $(X)$. A sample of each component (bole, branches and leaves) was obtained from each tree and both sample and total component fresh weight were determined in the field. The samples were taken to the laboratory to be dried and weighted so that, each tree's biomass could be estimated, using the dry weight to fresh weight ratios of the samples. To determinate carbon contents for the different components, samples were taken from 25 trees and analyzed in the laboratory to obtain carbon content for leaves of $52,21 \%$, branches $49,47 \%$, bole $49,26 \%$ and an average concentration $50,31 \%$. To estimate aboveground biomass the equation $B=0,0357{ }^{*} D N^{2.6916}$ was fitted and to estimate carbon content the equation fitted was $C C=0,021^{*} D N^{2.6451}$, with a coefficient of determination for both equations of 0,98 .
\end{abstract}

KEY WORDS:

allometry, biomass, carbon, equation, Pinus patula. México. ce: rosalino@correo.chapingo.mx

2 Instituto Nacional de Investigaciones Forestales, Agrícolas y Pecuarias. Campo Experimental Valle de México, km. 18.5, Carr. México-Lechería, AP 307, CP 56101, Texcoco, Edo. de México.

3 Colegio de Posgraduados, Carr. México-Texcoco km. 36.5, 56230, Montecillo, Texcoco, Edo. de México. 


\section{INTRODUCCIÓN}

El dióxido de carbono es uno de los gases de efecto invernadero que contribuye mayormente al calentamiento global, su contribución se ha calculado en 50 a $75 \%$, entre los gases traza; la concentración de este gas en la atmósfera ha aumentado de 280 ppm (partes por millón) en el año 1750, a 379 ppm en el 2004, y continúa incrementándose a una tasa anual mayor a 1 ppm (Castro et al., 2002; Epstein and Rogers, 2004; NETL, 2004). Ante la preocupación por el constante aumento de este gas, se han implementado diferentes estrategias para capturar el carbono y así reducir la concentración del $\mathrm{CO}_{2}$ en la atmósfera, ya sea a través de procesos bióticos o abióticos. Dentro del primer grupo comprende la fijación del $\mathrm{CO}_{2}$ como biomasa sobre la superficie terrestre, principalmente por las plantas que son las que lo utilizan en el proceso de la fotosíntesis. Respecto a la captura de carbono por procesos abióticos, consiste en la canalización de $\mathrm{CO}_{2}$ producido en las industrias o fábricas hacia las profundidades del océano o dentro del manto freático (Kimble et al., 2002).

La capacidad de fijación de carbono a través de procesos bióticos por los ecosistemas forestales aun es desconocida, ya que no se cuenta con procedimientos definidos para su estimación, se sabe que esta capacidad varía en función de la composición florística, la edad y la densidad de la población de cada estrato por comunidad vegetal (Schulze et al., 2000), sin embargo aun falta mucho por investigar respecto a esta variación.

En muchos estudios se ha empleado la biomasa de los árboles para estimar su contenido de carbono, a través de la multiplicación de la cantidad disponible en una determinada superficie por un factor que va desde 0,40 hasta el 0,55 , ya que varios autores han encontrado que es la proporción de carbono contenido en cualquier especie vegetal; Callo (2001), Fragoso (2003) y Zamora (2003) utilizaron 0,45, Barranco (2002), Herrera y Lucateros (2003) utilizaron 0,55 y Valenzuela (2001) utilizó 0,50, para determinar el carbono de varias especies vegetales. Por otra parte, Figueroa et al. (2005) determinaron porcentajes de carbono de $47,26 \%$ en encino (Quercus peduncularis) hasta $51,3 \%$ en el aile (Alnus glabrata), entre otras especies.

Para la estimación de la biomasa de un rodal forestal, que de acuerdo a Garzuglia y Saket (2003), quienes definen que "es la cantidad total de materia orgánica aérea presente en los árboles, incluyendo hojas, ramas, tronco principal y corteza", el procedimiento más común es el método de estimación por regresión. Este consiste en el muestreo destructivo de unos cuantos árboles para relacionar alguna de sus variables fáciles de medir con el contenido de biomasa, utilizando para ello métodos de regresión; por ejemplo, el diámetro normal (DN, diámetro a 1,30 m). Esta técnica es conocida como de análisis dimensional o alometría, lo cual consiste en el estudio del cambio de proporción de varias partes de un organismo como resultado de su crecimiento (López, 1988). Según Pastor et al. (1984), citados por Cano (1993), en estudios forestales el concepto de alometría se ha utilizado para relacionar componentes o variables de difícil medición (biomasa y área foliar), con variables más fáciles de medir (altura, diámetro, área basal y área de albura), con el objeto de estimar las primeras variables en función de las segundas; normalmente el parámetro del árbol que más se ha utilizado es el DN. Las ecuaciones de biomasa mejoran su predicción cuando se consideran al mismo tiempo las variables diámetro y la altura, pero la medición de alturas no se realiza en la práctica, debido a que toma 
mucho tiempo y en algunos bosques con coberturas cerradas no es fácil identificar la punta del árbol, sobre todo cuando las mediciones se hacen en forma indirecta. De cualquier manera, en la mayoría de los casos se logra una precisión satisfactoria cuando sólo se usa el DN como variable dependiente (Grier et al., 1977; Brown, 2001 y Acosta et al., 2002).

Con base en la problemática anterior, el presente estudio tuvo como objetivo generar dos ecuaciones alométricas: una para estimar la biomasa aérea total y otra para estimar el carbono aéreo total a partir del DN en Pinus patula Schl. et Cham., y de esta manera contribuir en la implementación de métodos de cuantificación de carbono capturado en la biomasa arbórea de esta importante especie forestal.

\section{MATERIALES Y MÉTODOS}

Los árboles empleados para generar la base de datos del presente estudio, fueron obtenidos de un predio bajo aprovechamiento en el Ejido Mariano de Matamoros, ubicado al Noroeste de la población El Rosario, municipio de Tlaxco, Tlaxcala. Geográficamente está situado entre las coordenadas 19\%40'15" y 1943'00" latitud Norte, 98 $12^{\circ} 49^{\prime \prime}$ y $98^{\circ} 15^{\prime} 12^{\prime \prime}$ longitud Oeste y a una altitud de 2,875 msnm (Fernández, 1998).

\section{Determinación de la biomasa}

Se seleccionaron 25 árboles de $P$. patula, sin deformidades ni enfermedades, los cuales ya estaban marcados para aprovechamiento; se trataron de incluir todas las categorías diamétricas que se encontraron en el predio. Antes de derribar cada árbol se midió su DN. Después de derribarlo, su fuste fue seccionado en trozas comerciales de 2,55 $\mathrm{m}$ de longitud las primeras, las últimas trozas fueron de $1,25 \mathrm{~m}$, que es el tamaño comercial de las trozas más pequeñas. Antes de medir cada troza comercial se obtuvo una rodaja de aproximadamente 5 $\mathrm{cm}$ de espesor, como muestra de cada troza. La primera se obtuvo de la sección que corresponde a la parte baja de la primera troza, las demás rodajas se obtuvieron de la parte más gruesa de las demás trozas (Figura 1). El procedimiento para la cubicación del tocón se realizó, como lo describe Avery y Burkhart (1983), esto es: comparar el volumen del tocón con el de un cono truncado o neiloide, que es lo recomendado para coníferas como la de este estudio. Las ramas fueron separadas del follaje y se pesaron de manera independiente, no sin antes haber obtenido de una a cuatro muestras de cada componente de acuerdo al tamaño del árbol. El peso fresco de cada componente, y su respectiva muestra se determinaron con una báscula de reloj de

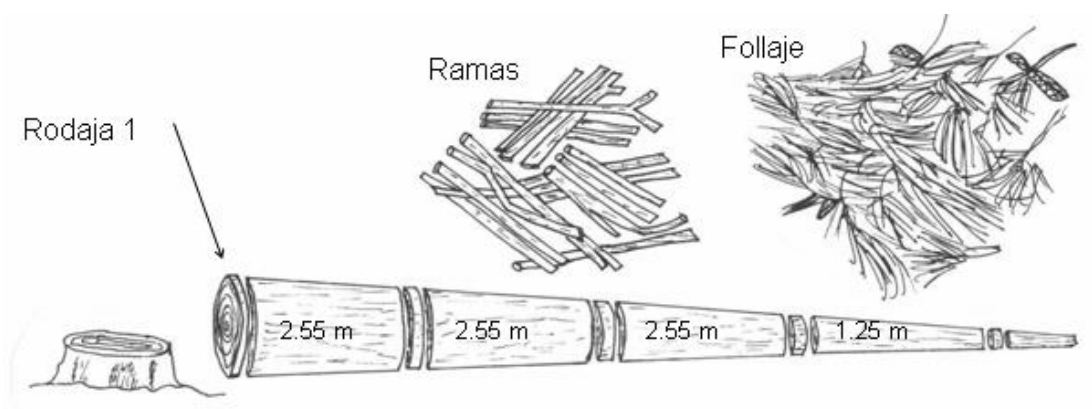

Figura 1.Troceo del arbolado, obtención de rodajas, muestras de ramas y follaje. 
$500 \mathrm{~kg}$ de capacidad, para pesar las trozas, las ramas y el follaje, y otra báscula de reloj de $15 \mathrm{~kg}$ de capacidad, para pesar las muestras de las trozas (rodajas) y las muestras de las ramas y el follaje. Toda esta actividad se realizaba en el lugar donde fue derribado cada árbol.

Los árboles pequeños $(6,0<\mathrm{DN}<10$ $\mathrm{cm})$ se cortaron y sin ser divididos en partes fueron transportados al laboratorio en donde fueron secados para obtener su biomasa, pesándolas directamente.

En total se obtuvieron 204 muestras del fuste (rodajas), 70 de ramas y 74 de follaje. Debido al gran volumen que representaron estas muestras, el secado en estufa no fue posible; por lo que se utilizó un microtúnel, a manera de estufa solar. El microtúnel tenía $3 \mathrm{~m}$ de ancho y $8 \mathrm{~m}$ de largo, y estaba cubierto con plástico. Para determinar el secado total del material se tomaron periódicamente muestras y se les determinó la humedad en estufa de secado. Cuando se observó que el peso seco registrado era constante, se procedió a pesar todas las muestras. EI peso de estos componentes ya secos se obtuvo utilizando una báscula que permitió pesar en gramos, para obtener los pesos con mayor precisión.

Para determinar la biomasa total de cada componente (fuste, ramas y follaje) del árbol, se empleó el factor resultante de la relación peso seco:peso fresco de la muestra tomada de cada componente y luego se multiplicó por el peso fresco correspondiente a todo el componente. La suma de la biomasa total del fuste (trozas) y de la copa (ramas, follaje), fue la biomasa aérea total de cada árbol (Acosta et al., 2002).

Una vez obtenidos los datos de biomasa aérea total y el DN de todos los árboles muestreados, se ajustaron los modelos (1) y (2), en su forma normal y en su expresión logarítmica, respectivamente. Este modelo se ha empleado en varios estudios para determinar la biomasa aérea de los árboles (Acosta et al., 2002; Figueroa, 2001; Gayoso et al., 2002; Schott, 2004).

$$
Y=b^{*} X^{k}
$$

donde $Y$ es la biomasa aérea $(\mathrm{kg}), X$ es el DN (cm), $b$ y $k$ son los parámetros de la función.

La expresión del modelo (1), en su forma lineal, mediante transformación logarítmica es la siguiente:

$$
\ln (Y)=\ln (b)+k \ln (X)
$$

donde $Y, X, b$ y $k$ tienen el mismo significado que en la ecuación (1), y In $(Y), \ln (b)$ y $\ln (X)$ son los logaritmos naturales de $Y, b$ y $X$, respectivamente.

Gayoso (2002), afirma que la preferencia por este modelo se debe a que expresa una proporcionalidad de los incrementos relativos entre dos componentes del árbol, y además es ampliamente consistente para distintas formas de crecimiento.

\section{Determinación del contenido de carbono}

De los 25 árboles seleccionados para la determinación de biomasa se eligieron cinco para determinar el porcentaje de carbono de cada uno de sus componentes. La selección de estos árboles fue equitativamente, dentro del rango de diámetros que se disponía (desde 6,7 a 64,1 cm).

De las muestras de cada componente arbóreo se tomó una submuestra de aproximadamente 50 gramos, que incluyó todo tipo de material que contenía 
la muestra, después éstos fueron molidos y enviados al Laboratorio de Fertilidad de Suelos del Colegio de Posgraduados de Montecillos, Estado de México, y el procedimiento que se siguió a cada submuestra para la determinación del carbono, fue el descrito por Acosta (2003).

Para estimar el contenido de carbono en la biomasa de los componentes de cada árbol se empleó la siguiente expresión:

$$
C C C=B T C * \% C
$$

donde CCC es el contenido de carbono por componente (fuste, ramas y follaje; expresado en $\mathrm{kg}$ ), BTC es la biomasa total del componente $(\mathrm{kg})$ y $\% C$ es el porcentaje de carbono del componente determinado directamente en el laboratorio.

El contenido del carbono de la biomasa total del árbol fue la suma del carbono del fuste, ramas y follaje de cada árbol, y para la determinación de la ecuación alométrica, para estimar el carbono, se empleó el modelo alométrico que ya se mencionó anteriormente (mismo que se ajustó a los datos de biomasa) y se correlacionó el contenido de carbono y el diámetro normal de cada árbol.

\section{RESULTADOS}

\section{Estimación de biomasa}

EI DN de los árboles muestreados varió en un rango de 6,7 a $64,1 \mathrm{~cm}$, y su biomasa de 7,62 a 2741,76 kilogramos. Los parámetros del modelo y la dispersión de puntos de los valores de biomasa observados se presentan en la figura 2. Al ajustar la ecuación para determinar la biomasa en función del diámetro normal presentó un coeficiente de determinación alto $\left(R^{2}=0,98\right)$.

La ecuación determinada para estimar la biomasa de $P$. patula Schl. et Cham, quedó expresada como:

$$
B=0,0357 \text { * } D N^{2.6916}
$$

en donde $B$ es la biomasa $(\mathrm{kg})$ y $D N$ es el diámetro normal $(\mathrm{cm})$.

\section{Estimación del carbono}

De los cinco árboles analizados, el porcentaje de carbono promedio para el componente fuste fue $49,26 \%$, con una desviación estándar de 0,46 con respecto a la media, para las ramas fue $49,47 \%$ y para el follaje $52,21 \%$, con desviaciones estándares de 1,32 y 2,31 , respectivamente (Tabla 1).

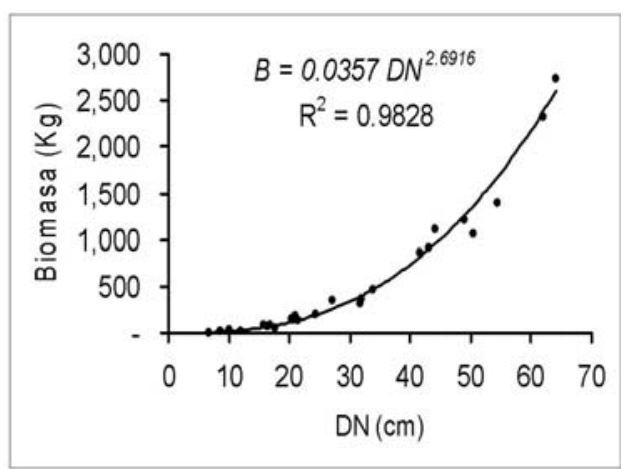

Figura 2. Dispersión de los valores observados de Pinus patula Schl. et Cham. y la línea de regresión generada con los datos de contenido de carbono. 
Tabla 1. Porcentaje de carbono por componentes de Pinus patula Schl. et Cham.

\begin{tabular}{|l|c|c|c|c|c|c|c|}
\hline Componentes & Árbol 1 & Árbol 2 & Árbol 3 & Árbol 4 & Árbol 5 & Promedio & Desv. Est. \\
\hline Fuste & 48,60 & 49,02 & 49,73 & 49,62 & 49,32 & $\mathbf{4 9 , 2 6}$ & 0,46 \\
\hline Ramas & 50,00 & 50,00 & 50,40 & 49,80 & 47,15 & $\mathbf{4 9 , 4 7}$ & 1,32 \\
\hline Follaje & 50,60 & 54,45 & 50,45 & 50,53 & 55,00 & $\mathbf{5 2 , 2 1}$ & 2,31 \\
\hline Árbol total & 49,73 & 51,16 & 50,19 & 49,98 & 50,49 & $\mathbf{5 0 , 3 1}$ & 0,55 \\
\hline
\end{tabular}

La ecuación para estimar el contenido de carbono quedó expresada como:

$$
C C=0,021 * D N 2.6451
$$

donde $C C$ es el contenido de carbono $(\mathrm{kg})$ y $D N$ es el diámetro normal (cm), la dispersión de puntos de los valores observados se presenta en la figura 3. Al ajustar la ecuación para determinar el contenido de carbono en función del diámetro normal también presentó un coeficiente de determinación alto $\left(\mathrm{R}^{2}\right.$ $=0,98)$.

Referente a la proporción de carbono por componente, el fuste almacena $82 \%$ del total, las ramas y el follaje, disponen del 10 y $8 \%$, respectivamente.

\section{DISCUSIÓN}

El coeficiente de determinación $\left(R^{2}\right)$ fue de 0,98 para ambas ecuaciones, lo cual significa que para estimar la biomasa y carbono de árboles de $P$. patula, es confiable la medición del DN. De la revisión de literatura se encontró que Castellanos (1993) determinó la biomasa de $P$. patula, para la región de ChignahuapanZacatlán, Puebla, tomando una muestra de 27 árboles, cuyos diámetros estuvieron en un rango de 5 a $45 \mathrm{~cm}$. Al comparar los resultados del presente estudio con los obtenidos por Castellanos, se encontró que las estimaciones de biomasa de Castellanos (1993) son mayores a las del presente estudio, en lo que comprende el rango de 5 a $30 \mathrm{~cm}$ de diámetro. Sin embargo, en los diámetros mayores de 30

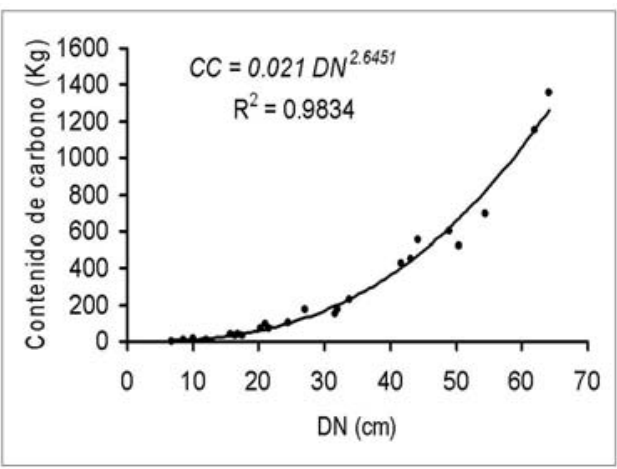

Figura 3. Dispersión y línea ajustada para los datos de contenido de carbono. 
cm la ecuación determinada en el presente estudio estima una biomasa mayor a la que se obtiene con la ecuación generada por Castellanos (Tabla 2). Esto puede atribuirse al rango de diámetros que manejó Castellanos (1993), que fue de 5 a $45 \mathrm{~cm}$, mientras que en el presente estudio se utilizaron árboles con diámetros de 6,7 a $64,1 \mathrm{~cm}$. En general, la variación en la estimación de biomasa no fue significativa, dado que se asemejan y se ubican dentro del intervalo de los valores de biomasa reportados para otras especies del género Pinus, estimados con el mismo modelo y considerando el DN como la variable independiente.

En la tabla 3 se muestran los resultados de estimación de biomasa para diferentes especies de pinos y diferentes estudios, donde se consideró calcular la biomasa de un árbol hipotético con un DN de $20 \mathrm{~cm}$. Los resultados variaron desde
87 kg para Pinus lambertiana, estudio realizado por Grier y Logan (1978), hasta 160 kg para Pinus virginiana, estudio realizado por Saucier y Boyd (1982).

En cuanto al contenido de carbono por componente, el follaje presentó 3\% más comparado con el fuste y las ramas. Sin embargo, la diferencia no fue significativa, por lo tanto es válido para este estudio y trabajos posteriores utilizar el $50,31 \%$ de la biomasa como el carbono total contenido en el arbolado de la especie $P$. patula.

\section{CONCLUSIONES}

Al ajustar el modelo $Y=b^{*} X^{k}$ a los datos de biomasa y carbono de $P$. patula, se determinaron parámetros con coeficientes de determinación $\left(\mathrm{R}^{2}\right)$ altamente confiables, mayores a 0,98.

Tabla 2. Biomasa para árbol total, obtenido del presente estudio y por Castellanos (1993).

\begin{tabular}{|c|c|c|}
\hline $\begin{array}{c}\text { Categoria Diamétrica } \\
(\mathrm{cm})\end{array}$ & $\begin{array}{c}\text { Biomasa total }(\mathrm{kg}) \\
\text { Presente estudio }\end{array}$ & $\begin{array}{c}\text { Biomasa total }(\mathrm{kg}) \\
\text { Castellanos }(1993)\end{array}$ \\
\hline 5 & 2,72 & 6,06 \\
\hline 10 & 17,55 & 29,38 \\
\hline 15 & 52,27 & 73,95 \\
\hline 20 & 113,38 & 142,37 \\
\hline 25 & 206,71 & 236,62 \\
\hline 30 & 337,67 & 358,37 \\
\hline 35 & 511,31 & 509,05 \\
\hline 40 & 732,44 & 989,91 \\
\hline 45 & 1005,67 & 1146,65 \\
\hline 50 & 1335,41 & 1424,53 \\
\hline 55 & 1725,95 & 1736,63 \\
\hline 60 & 2181,42 & 2083,77 \\
\hline 65 & 2705,86 & \\
\hline & & 902,09 \\
\hline
\end{tabular}


Tabla 3. Biomasa de árboles de Pinus spp. con diámetro de $20 \mathrm{~cm}$.

\begin{tabular}{|l|c|l|}
\hline \multicolumn{1}{|c|}{ Especie } & $\begin{array}{c}\text { Biomasa } \\
(\mathrm{Kg})\end{array}$ & \multicolumn{1}{|c|}{ Fuente } \\
\hline P. lambertiana & 87 & Grier y Logan, 1978. \\
\hline P. ponderosa & 79 & Gholz et al. 1979. \\
\hline P. virginiana & 160 & Saucier y Boyd, 1982. \\
\hline P. montezumae & 95 & Garcidueñas, 1987. \\
\hline P. patula & 142 & Castellanos, 1993. \\
\hline P. patula & 113 & Este estudio. \\
\hline
\end{tabular}

Dados los estimadores estadísticos se puede afirmar que las ecuaciones determinadas para estimar los valores de biomasa y carbono para $P$. patula son adecuadas y confiables para usarse en condiciones similares y para la misma especie.

El porcentaje de carbono en los árboles de $P$. patula fue de $50,31 \%$.

Del carbono total almacenado en árboles de $P$. patula, $5,07 \%$ está en el tocon, $78,82 \%$ en el fuste, $7,51 \%$ en las ramas y $8,6 \%$ en el follaje.

\section{REFERENCIAS}

Acosta-Mireles, M., J. Vargas-Hernández, A. Velásquez-Martínez y J.D. Etchevers-Barra. 2002. Estimación de la biomasa aérea mediante el uso de relaciones alométricas en seis especies arbóreas en Oaxaca, México. Agrociencia 36(6):725-736.

Acosta Mireles, M. 2003. Diseño y aplicación de un método para medir los almacenes de carbono en sistemas con vegetación forestal y agrícolas de ladera en México. Tesis de Doctorado en Ciencias. Colegio de Posgraduados. Montecillos, México. $135 \mathrm{p}$.
Avery, T.E. y H.E. Burkhart. 1983. Forest measurement. 3a. ed. McGraw-Hill Book Company, Nueva York. 331 p.

Barranco G., J. L. 2002. Captura de carbono retrospectivo, actual y potencial en el área natural protegida estatal Nahuatlaca-Matlazinca en el Estado de México. Tesis de Licenciatura. Universidad Autónoma Chapingo. Chapingo, México. 113 p.

Brown, S. 2001. Measuring carbon in forests: current status and future challenges. Environmental Pollution 116: 363-372.

Callo C., D. A. 2001. Cuantificación de carbono secuestrado por algunos sistemas agroforestales y testigos en tres pisos ecológicos de la amazonia del Perú. Tesis Maestría en Ciencias. Universidad Autónoma Chapingo. Chapingo Méx. 72 p.

Cano M., E. E. 1993. Relación alométrica entre el área foliar y el área de la albura en Pinus patula Schl. et Cham., en Zacatlán, Puebla. Tesis de Maestría en Ciencias. Colegio de Posgraduados. Montecillo, México. 89 p.

Castellanos B., J. F. 1993. Producción de biomasa y eficiencia de crecimiento 
en rodales de Pinus patula. Tesis de Maestría en Ciencias. Colegio de Posgraduados. Montecillos, México. $66 \mathrm{p}$.

Castro Z., R., R. Arteaga R., M. Vázquez P. y J. L. Jiménez R. 2002. Introducción a la meteorología. Universidad Autónoma Chapingo. México. 247 p.

Epstein, R. y C. Rogers. 2004. The impacts of $\mathrm{CO}_{2}$ and climate change on public health in the Inner City. The Center for Health and the Global Environment-Harvard Medical School.http://www.med.harvard.edu/ chge.

Fernández P., E. 1998. Programa de manejo forestal para el aprovechamiento de recursos forestales maderables para el "Ejido Mariano de Matamoros". Servicios Técnicos Forestales. Tlaxco, Tlaxcala. 54 p.

Figueroa N., C. M. 2001. Comparación de la concentración de carbono en diferentes tipos de vegetación de la Sierra Norte de Oaxaca. Tesis de Licenciatura. Universidad Autónoma Chapingo. Chapingo, México. 86 p.

Figueroa N., C., J. D. Etchevers B., A. Velásquez M. y M. Acosta M. 2005. Concentración de carbono en diferentes tipos de vegetación de la Sierra Norte de Oaxaca. Terra 23(1):57-64.

Fragoso L., P. I. 2003. Estimación del contenido y captura de carbono en biomasa aérea del predio "Cerro Grande" municipio de Tancítaro, Michoacán. Tesis de Licenciatura. Universidad Michoacana de San Nicolás de Hidalgo. Michoacán, México. 47 p.

Garcidueñas M., A.R. 1987. Producción de biomasa y acumulación de nutrientes en un rodal de Pinus montezumae Lamb. Tesis de Maestría. Colegio de Posgraduados. Programa Forestal. Montecillos, México. 242 p.

Garzuglia, M. y M. Saket. 2003. Wood volume and woody biomass. Food and Agricultural Organization of the United Nations. Roma. 30 p.

Gayoso, J. 2002. Medición de la capacidad de captura de carbono en bosques de Chile y promoción en el mercado mundial. Universidad Austral de Chile. Valdivia, Chile. $53 \mathrm{p}$.

Gholz, H.L., C.C. Grier, A.G. Campell y A.T. Brown. 1979. Equations and their use for estimating biomass and leaf area of Pacific Northwest planta. Res. Pap. 41. For. Res. Lab. Oregon State University. Corvallis, Oregon. $5 \mathrm{p}$.

Grier, C.C. y R.S. Logan, 1978. Oldgrowth Douglas-fir communities of a western Oregon watershed: biomass distribution and production budgets. Ecol. Monogr. 47(4):373-400.

Herrera D., V. F. y A. Lucatero B. 2003. Inventario de carbono capturado actual en el ejido Las Casitas municipio de Izúcar de Matamoros, Puebla, México. Tesis de Licenciatura. Universidad Autónoma Chapingo. Chapingo, México. 90 p.

Kimble, J. M., R. Lal y R. F. Follett. 2002. Agricultural practices and policies for carbon sequestration in soil. Lewis Publishers, EUA. 512 p.

López R., C. 1988. Modelos para estimación de biomasa de Pinus cembroides Zucc. Tesis de Licenciatura. Universidad Autónoma Chapingo. Chapingo, México. 55 p. 
NETL, 2004. Developing the technology base and infrastructure to enable sequestration as a greenhouse gas mitigation option. National Energy Technology Laboratory. Estados Unidos. Website: www.netl.doe.gov

Saucier J.R. y J.A. Boyd. 1982. Aboveground biomass of Virginia pine in North Georgia. U.S.D.A. Forest Service. Res. Pap. SE-232. Southeastern For. Exp. Sta. 32 p.

Schott, M. D. M. L. 2004. Determinación de los almacenes de carbono en sistemas de vegetación permanentes (forestal, café y acahual) en la Sierra Norte de Oaxaca. Tesis de Licenciatura. Universidad Autónoma Chapingo, Chapingo, México. 51 p.
Schulze, E. D., Ch. Wirt y M. Heimann. 2000. Managing forest after Kyoto. Science 289(5487):2058-2059.

Valenzuela H., T. 2001. Estimación de secuestro de carbono en bosques naturales de oyamel (Abies religiosa) en el sur del Distrito Federal. Tesis de Licenciatura. Universidad Autónoma Chapingo. Chapingo, México. 127 p.

Zamora C., J.C. 2003. Estimación del contenido de carbono en biomasa aérea en el bosque de pino del ejido "La Majada" municipio de Periban de Ramos, Michoacán. Tesis de Licenciatura. Universidad Michoacana de San Nicolás de Hidalgo. Michoacán, México. $47 \mathrm{p}$.

Manuscrito recibido el 11 de enero de 2006.

Aceptado el 31 de julio de 2006.

Este documento debe citarse como:

Díaz-Franco, R., M. Acosta-Mireles, F. Carrillo-Anzures, E. Buendía-Rodríguez, E. Flores-Ayala y J.D. EtcheversBarra. 2007. Determinación de ecuaciones alométricas para estimar biomasa y carbono en Pinus patula Schl. et Cham. Madera y Bosques 13(1):25-34. 